\title{
Segmentation of Road Guidance Sign Symbols and Characters based on Normalized RGB Chromaticity Diagram
}

\author{
Aryuanto Soetedjo \\ Dept. of Electrical Engineering \\ National Institute of Technology (ITN) \\ Malang Indonesia
}

\author{
Koichi Yamada \\ Dept. of MIS \\ Nagaoka University of Technology \\ Nagaoka Japan
}

\author{
F. Yudi Limpraptono \\ Dept. of Electrical Engineering \\ National Institute of Technology (ITN) \\ Malang Indonesia
}

\begin{abstract}
In this paper, we describe a color segmentation based on the normalized RGB chromaticity diagram to extract the symbols and characters of road guidance signs. The proposed method separates blue color of the signs by utilizing the developed histogram on the normalized RGB chromaticity diagram for selecting the threshold automatically. The image morphology operator and the histogram projection technique are employed to extract the symbols and characters. From the experiments on the real scene images, the extraction rate of $97.3 \%$ is achieved.
\end{abstract}

\section{General Terms}

Image processing, Machine vision.

\section{Keywords}

Color segmentation, normalized RGB chromaticity diagram, objects extraction, guidance signs.

\section{INTRODUCTION}

Nowadays, intelligent vision systems are growing rapidly. One of them is a system to detect and recognize road traffic signs using a camera installed in a vehicle. The system is a part of a driver assistance system (DAS), which assists a driver to recognize traffic signs when driving on the road.

In order to attract the drivers, traffic signs usually appear in colors contrast against the enviroments. Thus they are painted in blue, yellow, red, green and white colors. There are many types of traffic signs, such as: a) regulatory signs, eg. speed limit signs, no entry signs; b) indication signs, e.g. pedestrian crossing sign, parking sign; c) warning sign, e.g. road work sign; d) guidance sign, e.g. destination and route information sign. Each type is characterized by the shape, color, and symbols or characters painted on it. In the cases of regulatory, indication, and warning signs, the information could be intepreted easily by matching them to the reference signs, since they have a limited regular symbol. However, interpreting the information from the guidance sign is more difficult, because of the irregular symbols and the variation of characters. Thus it needs to extract the symbols and characters first before further process for intepreting them.

The first step to extract information in an image is image segmentation, which divides the image into non overlapping areas [1]. The area is a collection of pixels having the same characteristic, such as color, gray level, texture, etc. The color segmentation is a technique for separating objects according to the color. Many applications employ the color segmentation as frontend process for understanding an image, such in an interactive robot [2,3,4], video processing [5], traffic sign recognition $[6,7,8]$.

The common problem in the image processing for outdoor environment is the illumination changes that could not be controlled. Furthermore for real time implementation, it requires a fast algorithm. Therefore the color segmentation still becomes a challenging topic and offers the open area for improvement.

This paper addresses a color segmentation technique based on the normalized RGB chromaticity diagram. This proposed color segmentation technique is quite simple and effective to separate the blue color of the guidance sign background. Further, the morphology operation is employed to extract symbols and characters of the signs.

The rest of paper is organized as follows. In section 2, the related works are presented. The proposed method is covered in section 3. Section 4 covers the experimental results. Conclusion is described in section 5 .

\section{RELATED WORKS}

\subsection{Color Segmentation}

Color segmentation techniques could be classified into: histogram-based, boundary-based, region-based, and artificial intelligent-based.

The histogram-based method is usually used for grayscale image segmentation. Since a color image is represented in three components (RGB color), the histogram-based segmentation is performed by combining three thresholds obtained from each color channel. In [5], thresholding technique is carried out in each component of Red (R), Green (G), and Blue (B), to obtain the threshold values which are used as a base to produce a set of multithresholds used for video segmentation by unsupervised clustering.

In the boundary-based method, an edge detector is employed to find the boundary of an object. This method is based on the fact that intensity of the pixel will change rapidly in the boundary of two regions. For the color segmentation, the edge detection is employed for each color channel (R,G,B), then the resulted edges are fused to obtain the final edge image.

In the region-based method, pixels are grouped according to the homogenity criteria. The region growing and split and merge algorithms are the examples of the region-based method. In the region growing algorithm, pixels or subregions are grouped into larger regions based on predefined criteria [9]. The algorithm starts with a set of seed points and then grows regions by 
appending to each seed those neighboring pixels that have similar properties to the seed, such as gray level or color. On the contrary, the split and merge algorithm subdivide an image initially into a set of arbitrary, disjointed regions and then merge and/or split the regions in attemp to satisfy the predefined criteria [9]. These techniques have two main drawbacks [1]: They are both strongly dependent on global predefined criteria; while the region growing technique depends also on initial segments, which is the first pixel/segment to be scanned, and the order of the process.

In [10], the multilayer perceptron (MLP) network is adopted for adaptive color image segmentation. They use multisigmoid activation function for segmentation. The number of steps i.e. thresholds in the multisigmoid function are dependant on the number of clusters in the image, which are found automatically from the first order derivative of histograms of saturation and intensity in the HSV color space.

Fuzzy logic model that follow the human intuition for color classification is proposed in [11]. In this approach, fuzzy sets are defined on the $\mathrm{H}, \mathrm{S}$, and V components of the HSV color space and divides the color space into segments based on linguistic terms. The fuzzy rules are defined based on human observation to classify the color produced by the tree components of the HSV color space.

Genetic Algorithm (GA) is adopted in [12] to optimize the color segmentation. The process evolves in a sequence of steps, where at each step a GA optimizes the previous segmentation results until a satisfactory segmentation is achieved. The GA starts from an initial random population of $\mathrm{N}$ individuals, then it performs $\mathrm{N}$ segmentations of the image according to the parameters of each individual. Then a fitness is evaluated for each resulting image, and the GA cycles, i.e. genetic operators (selection, crossover, mutation) are applied on the processing parametes of these individuals until the fitness does not exceed a given threshold or a maximum number of iterations is reached.

\subsection{Extraction of Guidance Sign Symbols and Characters}

A guidance sign as illustrated in Figure 1 is used to give traffic information to the users (drivers or pedestrians). It is characterized by its rectangular shape and blue color with white characters and symbols. Since color contains useful information, many researchers $[6,7,8]$ prefer to use color segmentation to extract the symbols and character from the sign images.

In [6], a blue color segmentation using LUV ( $\mathrm{L}$ is luminance, $\mathrm{U}$ and $\mathrm{V}$ are chrominance components) color space is taken to extract the blue color from an image and then finds the edge of rectangular shape to identify the guidance sign. To overcome the problem of illumination changes, they employ a multiplethresholds approach. The thresholds are determined by analyzing the actual distribution of data in the YUV color space taken in various weather and lighting conditions. The detected sign consists of two colors (blue and white), results in two clusters having the highest value in the intensity histogram. Then, characters and symbols are extracted by transformation of intensity histogram using the following equation:

$$
I_{\text {new }}=\left\{\begin{array}{lrr}
255 \frac{I_{\text {org }}-I_{\min }}{I_{\max }-I_{\min }} & \text { if } & I_{\min } \leq I_{\text {org }} \leq I_{\max } \\
0, & \text { otherwise }
\end{array}\right.
$$

where $I_{\min }$ and $I_{\max }$ are the minimum and maximum intensity of the cluster in the histogram, respectively. $I_{\text {org }}$ is intensity of the original image and $I_{n e w}$ is the intensity of transformed image. Using this intensity transformation, characters and symbols could be extracted fairly stably using one threshold in various lighting conditions. This similar approach is also adopted in [7].

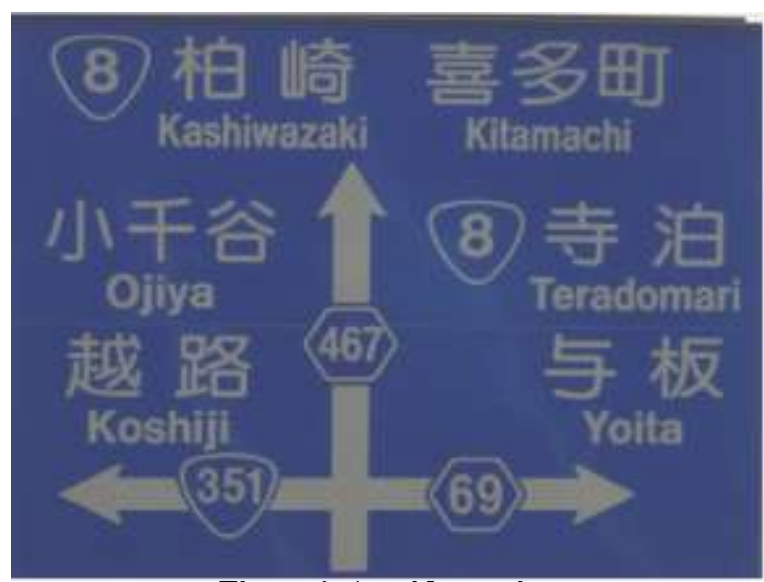

Figure 1. A guidance sign.

After the image transformation process as desrcribed above, the edge detection and image morphology operation are employed to fill the existing holes. Since the area of symbols, i.e. the arrows usually are large, then the symbols are separated from the characters by the number of pixels. The position of characters is defined from the projection to the vertical axes to produce the histogram. The dominant peaks in the histogram define the vertical positions of the characters in the guidance signs.

In [8], to localize road sign (road sign that contains text) from an image, $H$ component of HSI color space is used for color modeling and $k$-mean algorithm is applied for clustering. Using this method, all red points (pixels) are in one cluster, the same for blue, green and yellow ones. Then, for extracting texts in the sign, color modeling of background and foreground (text) of detected road sign is applied.

The other works related to the characters extraction are conducted in [12] to find the characters in the image, and in [13] to help the visual impaired people recognizes the signs.

The clustering technique based on the normalized RGB is used to separate the color the homogen color layers [12]. Then the connected component analysis is performed for all color layers, and the bounding boxes of the components are defined. Finally, the characters are identified by a heuristic approach.

In [13], the Otsu thresholding technique is employed on three color channels to extract the characters. To avoid the misdetection, a selection rule based on the relative position of the connected components is employed. 


\section{PROPOSED METHOD}

In our previous works $[14,15]$, we proposed a color segmentation technique based on the normalized RGB chromaticity diagram for detecting red color sign [14] and detecting human skin color [15]. Here we extend the approach to detect the blue color as the front end process to extract the symbols and characters of the guidance signs.

The normalized RGB chromaticity diagram is depicted in Figure 2 , where the chromaticity coordinates are $r$ and $g$ defined by

$$
\begin{gathered}
r=\frac{R}{R+G+B} \\
g=\frac{G}{R+G+B}
\end{gathered}
$$

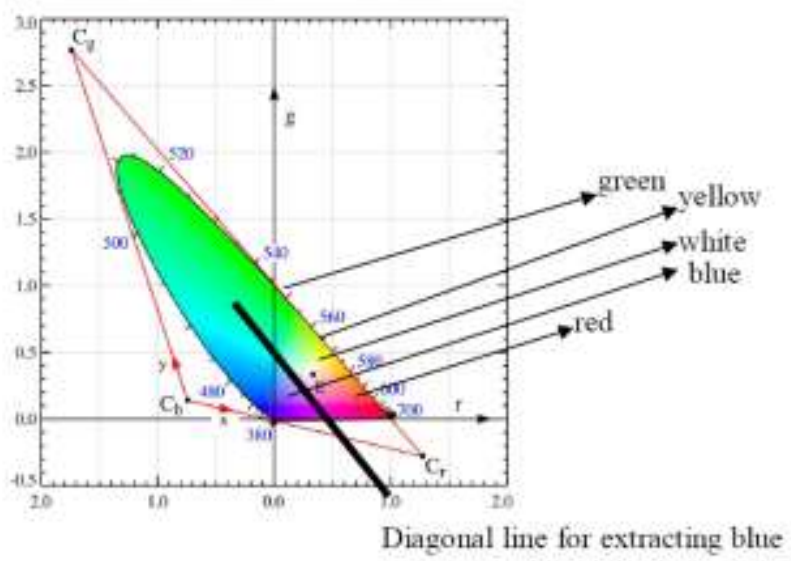

Figure 2. Normalized RGB chromaticity diagram.

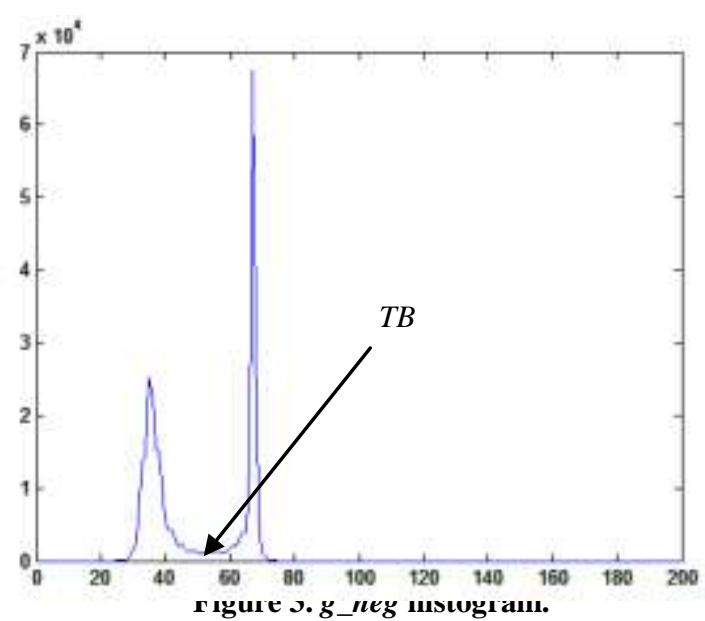

The diagonal line for extracting blue color is shown in the Figure 2. This line is determined by the following equation

$$
g=-r+T B
$$

where $T B$ is the intersection of the line with $g$-coordinate. $T B$ is calculated automatically by analyzing the peaks/valleys of the newly developed histogram called $g_{-}$neg histogram [15]. The g_neg histogram is created by counting pixels with the value obtained by adding $g$ value and $r$ value $(g+r)$. The effectiveness of the histogram is that it shows prominent peaks/valleys for easy threshold calculation. Thus by employing histogram peaks/valleys analysis, we may find the appropriate diagonal line for separating blue color. Figure 3 shows the $g \_n e g$ histogram with the prominent peaks, where the valley of histogram that determines the value of $T B$ might be obtained easily.

From Figure 2, it is clear that the blue color could be extracted by the following rule:

$$
\text { If } g+r<T B \text { then pixel is BLUE }
$$

The guidance sign as illustrated in Figure 1 contains three kinds of information, i.e. the symbol indicating the road structure (represented by the arrows), characters (Kanji and/or Alphabet) indicating city or location, and numbers indicating the route numbers. In this research, we extract the symbols, characters, and numbers, while in [6] they only extract the symbols and characters.

Our proposed method starts with extracting the route numbers. From the observation of Figure 1, we could see that the route numbers are located inside small polygons with blue color background and white color in the boundary. Thus, if we employ the blue color segmentation on the guidance sign image, two kinds of objects with blue color backgrounds will be extracted, i.e. the background of a whole image and the background of small polygons containing the route numbers. The small polygons could be separated from the whole sign background by applying a threshold to the numbers of pixels of the extracted blue color objects.

In this research, we normalize the size of the guidance sign image into 640 X 480 pixels. Thus, the extracted blue color objects are classified as the small polygons if the numbers of blue pixels are greater than 500 pixels and lower than 5000 pixels. Since, the color of numbers inside the polygons are white, there will be holes inside the polygons. To obtain the solid regions, we apply the morphology operator: dilation and hole-filling. Figure 4 shows the extracted regions of the route numbers of the image in Figure 1. Figure $4(a)$ is the resulted image after the blue color segmentation, where the extracted blue color objects are drawn in white color. While the black color objects in Figure 4(b) represent the regions of the route numbers.

The second step is extraction of the symbols (arrow symbols). Since the area of symbols painted with the while color are larger than the other white objects (characters, numbers, and small polygons), the symbols are extracted using the criteria of the numbers of white pixels similar to the method in [6]. However, this method has a drawback as follows. In some cases the extracted characters are connected with the neighbor, thus form a large area of the connected component. The similar problem also occurs in the boundary of polygons which are connected with the arrows. To overcome the problems, we employ the morphology operation- erosion to dismiss the non arrow objects as illustrated in Figure 5(a). Then the symbols or arrows are extracted if the numbers of pixels of the connected component (black pixels in Figure 5(a)) are greater than 1000 pixels.

After two steps described above are performed, we have the remaining objects of the characters (Kanji and Alphabet) as 
shown in Figure 5(c). Therefore, the next step is extracting those characters. To extract the characters, we employ the histogram projection technique [6] to define the regions of the characters. If image in Figure 5(c) is projected to the vertical axes, we obtain the histogram which the peaks denote the vertical position of the characters as shown in Figure 6(a). The extracted characters (regions of characters) are shown in Figure 6(b).

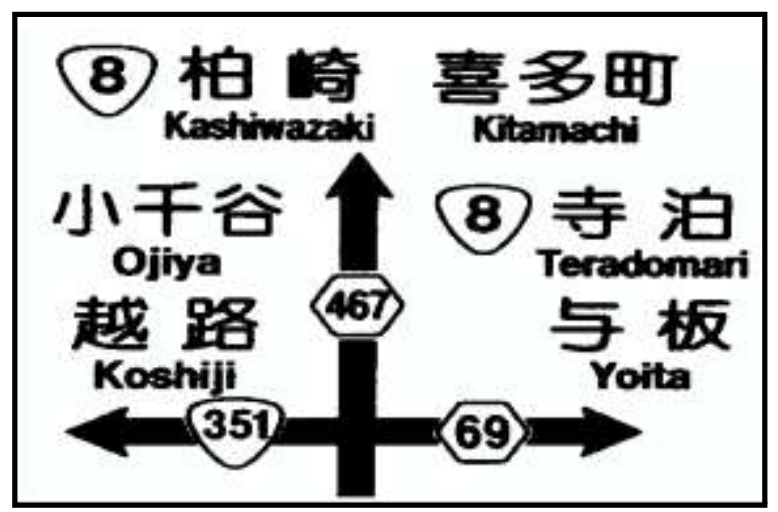

(a)

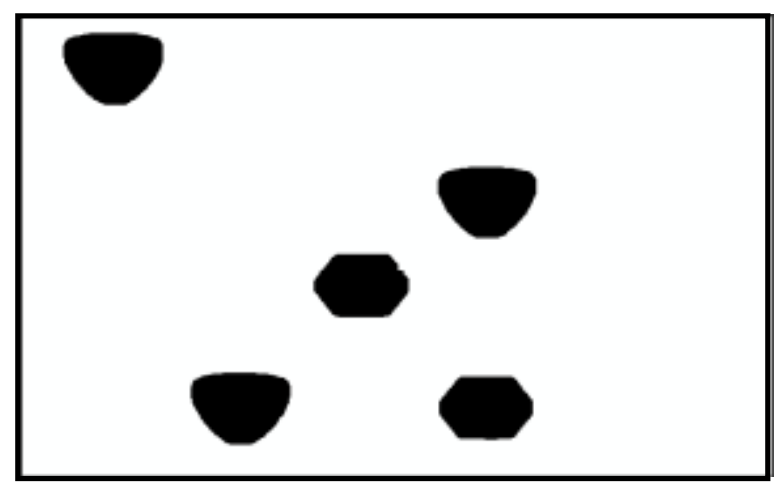

(b)

Figure 4. (a) Result of the blue color segmentation; (b) The extracted regions of the route numbers.

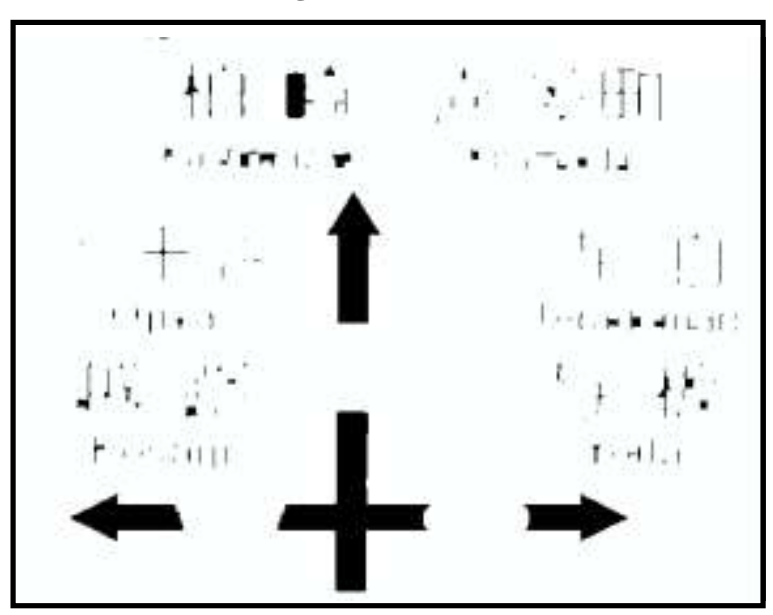

(a)

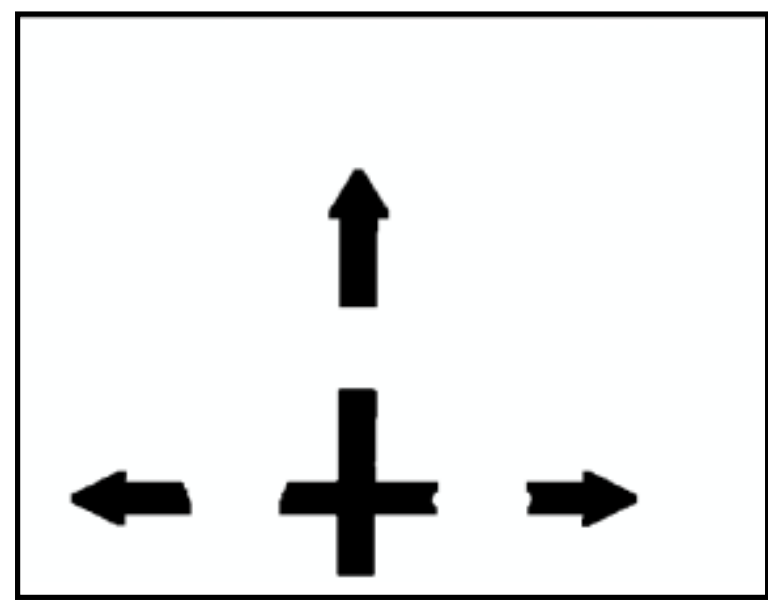

(b)

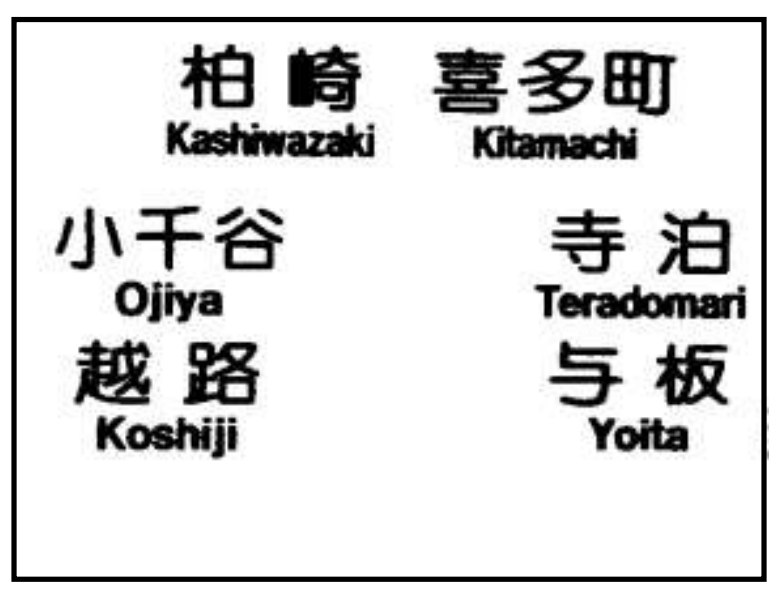

(c)

Figure 5. (a) Result of erosion; (b) The extracted symbols; (c) The extracted Kanji and Alphabet.

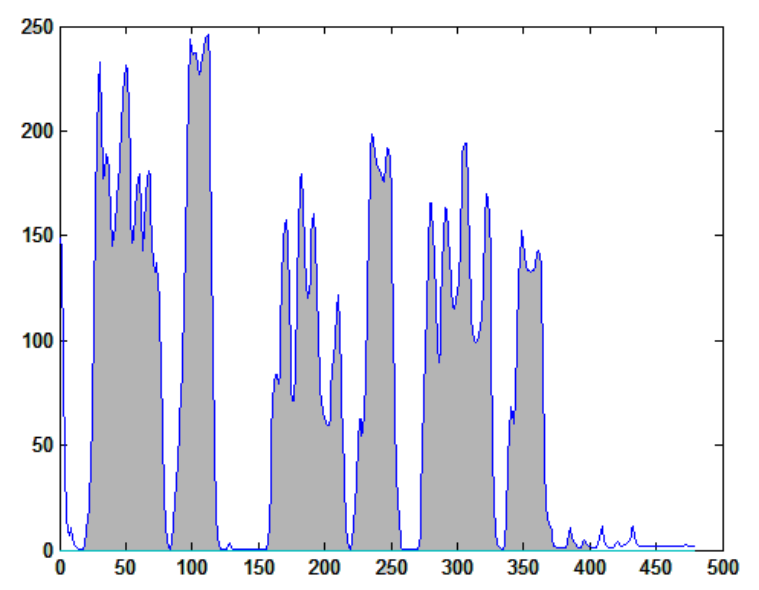

(a) 


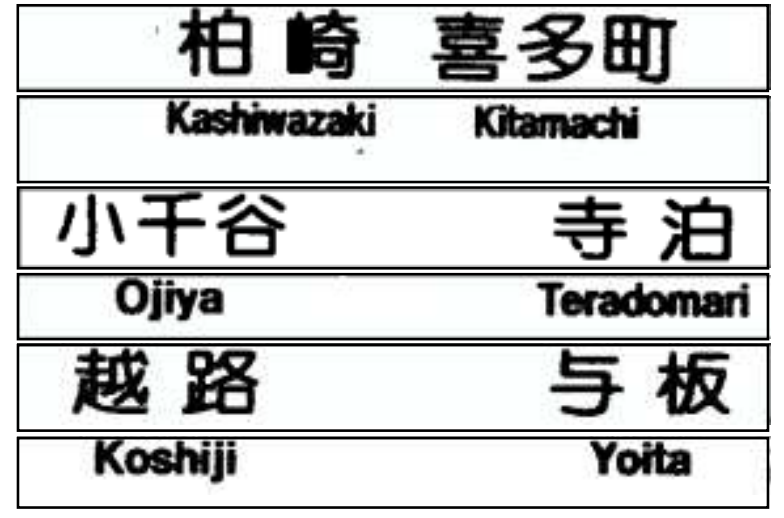

(b)

Figure 6. (a) Histogram projection on the vertical axes; (b) The extracted regions of the characters.

\section{EXPERIMENTAL RESULTS}

To verify our method, we test our algorithm using real scene images taken from a camera. The guidance sign images are collected from fifteen (15) different locations. The algorithm is implemented using MATLAB running on a PC.

For comparison, we also test the method in [6] using the same images. Table 1 shows the experimental results. Since in [6], they do not extract the route numbers, the total extraction rate is calculated from the averages of the symbols and characters extraction rates.

The extraction rate of the route numbers is calculated from dividing the total numbers of the extracted route numbers by the total numbers of the route numbers in the sign images. The extraction rate of the symbols is calculated from dividing the total numbers of the extracted symbols by the total numbers of the symbols in the sign images. The extraction rate of the characters is calculated from dividing the total numbers of the extracted row regions contain the characters by the total numbers of the row regions contain the characters in the sign images. The row regions contain Kanji and Alphabet are calculated separately.

From our experiments, we find that error in the characters extraction is usually caused by the arrangement of the characters (Kanji and Alphabet) which occupy in the overlapping rows as shown in Figure 7(a). From the histogram projection shown in Figure 7(b), it is clear that we could not find the valleys for separating the row regions.

Table 1. Experimental results.

\begin{tabular}{|l|c|c|}
\hline & Proposed method & Method in [6] \\
\hline $\begin{array}{l}\text { Extraction rate } \\
\text { of the route } \\
\text { numbers }\end{array}$ & $100 \%$ & - \\
\hline $\begin{array}{l}\text { Extraction rate } \\
\text { of the symbols }\end{array}$ & $100 \%$ & $87 \%$ \\
\hline $\begin{array}{l}\text { Extraction rate } \\
\text { of the characters }\end{array}$ & $92 \%$ & $78 \%$ \\
\hline $\begin{array}{l}\text { Total extraction } \\
\text { rate }\end{array}$ & $97.3 \%$ & $82.5 \%$ \\
\hline
\end{tabular}

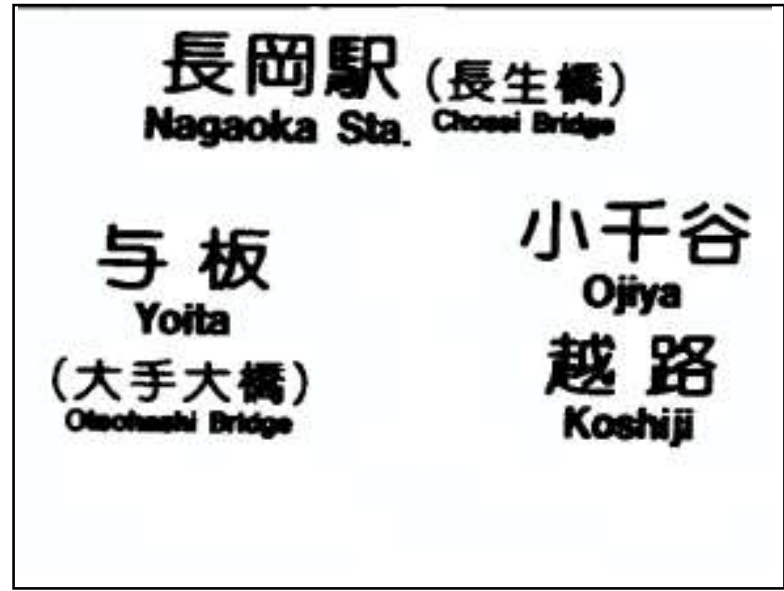

(a)

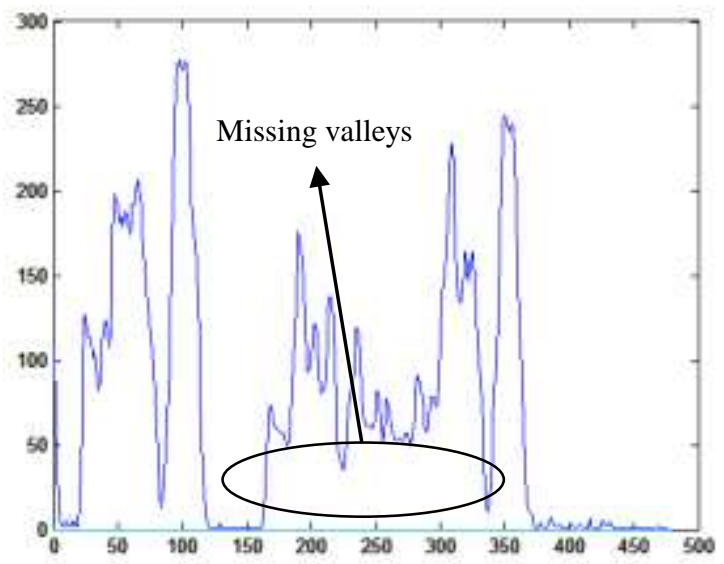

(b)

Figure 7. (a) The characters in overlapping row regions; (b) Missing valleys in the histogram projection.

\section{CONCLUSION}

In this paper, a color segmentation technique for extracting symbols and characters of road guidance sign image is presented. The method is based on the normalized RGB chromaticy diagram. The experiments shows that the method achives a high extraction rate.

In future, we will extend the work for recognizing or interpreting the extracted symbols and characters.

\section{REFERENCES}

[1] Navon, E., Millier, O. , Averbuch, A. 2005.Color Image Segmentation Based on Adapative Local Thresholds, Image and Vision Computing, Vol. 23, pp. 69-85.

[2] Bruce, J., Balch, T. , Veloso, M. 2000. Fast and Inexpensive Color Image Segmentation for Interactive Robots, Proc. of International Conference on Intelligent Robots and System, Takamatsu, Japan, Oct 2000, pp. 2061-2066.

[3] Browning, B., Veloso, M. 2005. Real-time, Adaptive Colorbased Robot Vision, Proc. of International Conference on 
Intelligent Robots and System, Edmonton, Canada, Aug 2005, pp. 3871- 3876.

[4] Li, B., Hu, H., Spacek, L. 2003.An Adaptive Color Segmentation Algorithm for Sony Legged Robots, Proc. of the $21^{\text {st }}$ IASTED International Conference Applied Informatics, Innsbruck, Austria, Feb. 2003, pp. 126 -131.

[5] Du, Y. Z., Chein-I, C., David, T.P. 2004. Unsupervised Approach to Color Video Thresholding, Optical Engineering, Vol. 43, No. 2, pp. 282-289.

[6] Miura, J., Kanda, T., Shirai, Y. 2000. An Active Vision for Real Time Traffic Recognition, Proc. of IEEE Conference on Intelligent Transportation Systems, Dearborn, MI, pp. 52-57.

[7] Lee, J.H., Jo, K.H. 2003. Traffic Sign Recognition by Division of Characters and Symbols Regions, Proc. of the $7^{\text {th }}$ Korea-Russia International Symposium, KORUS.

[8] Wu, W., Chen, X., Yang, J. 2004. Incremental Detection of Text on Road Signs from Video with Application to a Driving Assistant System, Proc. of ACM Multimedia 2004, New York, USA.

[9] Gonzalez, R.C., Woods, R.E. 2002. Digital Image Processing, Prentice Hall.

[10] Shinde, G.N., Deshmukh, K.S. 2005. An Adaptive Color Image Segmentation, Electronic Letter on Computer Vision and Image Analysis, Vol. 5, No. 4, pp. 12-23.
[11] Shamir, L. 2006. Human Perception-based Color Segmentation Using Fuzzy Logic, Proc. of International Conference on Image Processing, Computer Vision and Pattern Recognition (IPCV 2006), Las Vegas, USA, June 2006, pp. 496-505.

[12] Wang, K., Kangas, J.A. 2003. Character Location in Scene Images from Digital Camera, Pattern Recogntion, Vol. 36, pp. 2287-2299.

[13] Ezaki, N., Kiyota, K., Minh, B.T. Bulacu, M. Schomaker, L. 2005. Improved Text-Detection Methods for a Camera-based Text Reading System for Blind Persons, Proc. of $8^{\text {th }}$ International Conference on Document Analysis and Recognition (ICDAR'05)”, pp. 257-261.

[14] Soetedjo, A., Yamada, K. 2007. A New Approach on Red Color Thresholding for Traffic Sign Recognition System, Journal of Japan Society for Fuzzy Theory and Intelligent Informatics, Vol. 19, No. 5, pp. 457-465.

[15] Soetedjo, A., Yamada, K. 2008. Skin Color Segmentation Using Coarse-to-Fine Region on Normalized RGB Chromaticity Diagram for Face Detection, IEICE Trans. On Information and Systems, Vol. E91-D, No. 10, pp. 24932502 . 